\title{
Raman and TEM studies of Ge nanocrystal formation in $\mathrm{SiO}_{\mathrm{x}}: \mathrm{Ge} / \mathrm{SiO}_{\mathrm{x}}$ multilayers
}

\author{
A. Dana ${ }^{*}, 1$, S. Ağan ${ }^{2}$, S. Tokay ${ }^{2}$, A. Aydınlı ${ }^{1}$, and T. G. Finstad ${ }^{3}$ \\ ${ }^{1}$ Physics Department and National Nanotechnology Research Center, Bilkent University, 06800 Ankara, \\ Turkey \\ 2 Physics Department, Kırıkkale University, 71450 Kırıkkale,Turkey \\ 3 Physics Department, University of Oslo, P.O. Box 1048 - Blindern, 0316 Oslo, Norway
}

Received 30 July 2006, revised 3 September 2006, accepted 3 September 2006

Published online 7 February 2007

\section{PACS 68.37.Lp, 68.65.-k, 81.07.Bc, 81.15.Gh, 81.40.Ef}

\begin{abstract}
Alternating germanosilicate-siliconoxide layers of 10-30 nm thickness were grown on Si substrates by plasma enhanced chemically vapor deposition (PECVD). The compositions of the grown films were determined by X-ray photoelectron spectroscopy measurements. The films were annealed at temperatures varying from 670 to $1000{ }^{\circ} \mathrm{C}$ for 5 to 45 minutes under nitrogen atmosphere. High resolution cross section TEM images, electron diffraction and electron energy-loss spectroscopy as well as energy-dispersive $\mathrm{X}$-ray analysis (EDAX) data confirm presence of Ge nanocrystals in each layer. The effect of annealing on the Ge nanocrystal formation in multilayers was investigated by Raman spectroscopy and Transmission Electron Microscopy (TEM). As the annealing temperature is raised to $850{ }^{\circ} \mathrm{C}$, single layer of Ge nanocrystals observed at lower annealing temperatures is transformed into a double layer with the smaller sized nanocrystals closer to the substrate $\mathrm{SiO}_{2}$ interface.
\end{abstract}

() 2007 WILEY-VCH Verlag GmbH \& Co. KGaA, Weinheim

1 Introduction The possible use of semiconductor nanocrystals for applications in electronic and optical devices has provided strong motivation for research in this field. Despite the indirect band gaps associated with bulk silicon and germanium inhibiting light emission, both quantum confinement effects and their compatibility with current Si based microelectronics process technology makes Si and Ge nanocrystals even more attractive. Many research groups have studied the formation and subsequent optical and electrical properties of Si and Ge nanocrystals [1]. Among many properties of interest, structural information revealing crystal structure, nanocrystal shape, type and density defects is fundamental because they determine almost all other properties of the nanocrystals. Structural information can be obtained by several analytical techniques such as X-ray diffraction (XRD), transmission electron microscopy (TEM) and Raman scattering spectroscopy to name a few [2,3]. Ge nanocrystals have been obtained by ion beam synthesis in $\mathrm{SiO}_{2}$ and post growth annealing $[4,5]$. TEM characterization showed $\mathrm{Ge}$ nanocrystals with a mean diameter of few nm's depending on implantation dose and annealing time and temperature.

In this work, we report on formation of Ge nanocrystals in multilayered grown with plasma enhanced chemical vapor deposition (PECVD). We used both high resolution TEM and Raman scattering spectroscopy to determine size and crystallinity of Ge nanocrystals. The size dependent spatial separation of Ge nanocrystals has been observed. This may be crucial for memory device applications. PECVD allows for the control of the thickness of the $\mathrm{SiO}_{2}$ tunnel oxide underneath the nanocrystal layer, as well as the density and size of the Ge nanocrystals.

\footnotetext{
* Corresponding author: e-mail: sedatagan@kku.edu.tr, Phone: +00 90318 3572478, Fax: +00 903183572461
} 
2 Experimental procedure The oxide $(10 \mathrm{~nm})$-germanosilicate $(10-30 \mathrm{~nm})$ - oxide $(50 \mathrm{~nm})$ multilayered films were grown in a PECVD reactor (model PlasmaLab 8510C) on Si substrates using 180 sccm $\mathrm{SiH}_{4}\left(2 \%\right.$ in $\left.\mathrm{N}_{2}\right), 225 \mathrm{sccm} \mathrm{N} \mathrm{N}_{2} \mathrm{O}$ and varying flow rates of $\mathrm{GeH}_{4}(2 \%$ in $\mathrm{He})$ as precursor gases, at a sample temperature of $350^{\circ} \mathrm{C}$, a process pressure of 1000 mTorr under and an applied RF power of 10 W. First, a tunnel oxide followed by a deposition of a $\mathrm{SiO}_{\mathrm{x}}$ :Ge layer and a layer of $\mathrm{SiO}_{\mathrm{x}}$ was grown. $\mathrm{SiO}_{\mathrm{x}}: \mathrm{Ge}$ and $\mathrm{SiO}_{\mathrm{x}}$ layers form a pair. Samples with layers ranging from 3 to 10 pairs have been grown. Germanium doped $\mathrm{SiO}_{\mathrm{x}}: \mathrm{Ge}$ layer is where nanocrystals form upon annealing. The samples were then annealed in $\mathrm{N}_{2}$ atmosphere in a quartz oven at temperatures ranging from 670 to $1000{ }^{\circ} \mathrm{C}$ for 5 to 45 minutes. The structural characterization was carried out in a $200 \mathrm{kV}$ JEOL $2010 \mathrm{~F}$ field-emission TEM located at University of Oslo, making use of diffraction, tilt and rotation facilities.

3 Results and discussion The samples were grown as alternating layers of germanosilicate and silicon oxide using PECVD. Deposited layers are prepared by varying the flux ratios of $\mathrm{SiH}_{4}\left(2 \%\right.$ in $\left.\mathrm{N}_{2}\right)$ and $\mathrm{GeH}_{4}(2 \%$ in $\mathrm{He})$, systematically. These have been heat treated at different temperatures 670 to 900 ${ }^{\circ} \mathrm{C}$ for 5 to $45 \mathrm{~min}$. Cross-sectional TEM image in Fig. 1 shows 3 germanosilicate layers with nanocrystal formation annealed at $900{ }^{\circ} \mathrm{C}$ for $45 \mathrm{~min}$. First layer consists of $12 \mathrm{~nm}$ thick germanosilicate and the

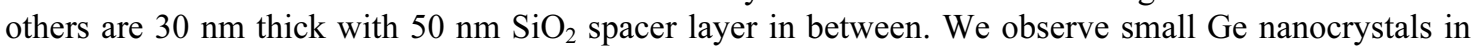
the first germanosilicate layer at the $\mathrm{SiO}_{2} / \mathrm{Si}$ interface while $\mathrm{Ge}$ nanocrystals in the second and third layers are larger. Smaller nanocrystals are due to limited amount of Ge in the thinner first germanosilicate layer. Based on HRTEM images of these samples, Ge nanocrystal sizes range 7-30 nm. The micrograph shows perfect alignment of crystallographic planes in the Ge nanocrystals that have clearly pronounced facets. From the figures, different nanocrystals show different orientations of crystallographic planes. We see the formation of Ge nanocrystals in each layer of the multilayer sample. Ge particles were analyzed with selected area diffraction (SAD). Figure 2(a) shows a typical diffraction pattern with rings indicating randomly oriented diamond Ge crystals. Table 1 summarizes average lattice parameters. The particle sizes increase as function of annealing temperature. Figure 2(b) shows nanoparticle size distribution in the layers with the largest $\mathrm{Ge}$ concentration and annealing at $770{ }^{\circ} \mathrm{C}$ for $5 \mathrm{~min}$.

Figure 3 displays the results of Raman measurements from the same samples displaying the evolution of Ge nanocrystal formation upon annealing at temperatures in the range of $600-900^{\circ} \mathrm{C}$. As an example, we show the spectra for samples in the annealing temperature ranges 600 to $100{ }^{\circ} \mathrm{C}$ for $45 \mathrm{~min}$. (Fig. 3(a)). The spectrum remains unchanged for the annealing temperatures less than $600{ }^{\circ} \mathrm{C}$. We observe a very broad $\left(\sim 40 \mathrm{~cm}^{-1}\right)$ asymmetric peak centered around $291 \mathrm{~cm}^{-1}$ indicative of the quasi amorphous nature of the Ge for samples annealed at $600{ }^{\circ} \mathrm{C}$. Si substrate is observed at $520.4 \mathrm{~cm}^{-1}$. If the annealing temperature is raised to $700{ }^{\circ} \mathrm{C}$, a sharp peak at $299 \mathrm{~cm}^{-1}$, now $10 \mathrm{~cm}^{-1}$ in width, is accompanied by a wide shoulder on the low frequency side. The sharp peak is a clear sign of Ge nanocrystal formation accompanied by a range of smaller Ge nanostructures. We note that this peak is at a lower frequency than the Ge mode in bulk Ge. This is due to phonon confinement in small crystals [6]. This peak becomes stronger and narrower $\left(8 \mathrm{~cm}^{-1}\right)$ and the broad quasi amorphous structure becomes weaker as the annealing temperature is increased up to $800{ }^{\circ} \mathrm{C}$. At this temperature, in addition to the features associated with $\mathrm{Ge}$ nanocrystals, the spectrum now displays a weak but clearly discernible asymmetric peak centered at $419.5 \mathrm{~cm}^{-1}$ indicating the formation of SiGe alloy at the oxide Si substrate interface. In fact, if the oxide layer is completely removed with dilute HF solution, the same board peak at $419.5 \mathrm{~cm}^{-1}$ is still observable. Furthermore, remnants of the quasi amorphous feature completely disappear in favor of an asymmetric Ge mode which now shifts to $304 \mathrm{~cm}^{-1}$. The linewidth of the $\mathrm{Ge}$ mode increases dramatically when the sample is annealed at $900{ }^{\circ} \mathrm{C}$. The SiGe alloy peak is stronger at $413 \mathrm{~cm}^{-1}$, also asymmetric on the low frequency side. Finally, the Si substrate peak observed as a sharp peak centered about $520.5 \mathrm{~cm}^{-1}$ is accompanied by a broad shoulder on the low frequency side centered around $499 \mathrm{~cm}^{-1}$. This is $\mathrm{Si}-\mathrm{Si}$ appearing due to further interdiffusion of $\mathrm{Si}$ and $\mathrm{Ge}$ at the interface. With increasing annealing temperature, these peaks behaved differently. A strong Si optic mode from the substrate is observed at $520 \mathrm{~cm}^{-1}$. The annealing temperature dependence of the Raman spectrum depends on the Ge concentration in films. 
We found that as the Ge concentration decreases, annealing at a higher temperature is necessary to obtain samples showing the sharp Ge mode in the Raman spectrum.

4 Conclusions We have shown the formation of crystalline Ge nanocrystals in $\mathrm{SiO}_{2}$ matrices grown with PECVD technique by using HRTEM, EDAX, Raman scattering and electron diffraction. Each layer in the multilayer of $\mathrm{SiO}_{\mathrm{x}}: \mathrm{Ge} / \mathrm{SiO}_{\mathrm{x}}$ system contains $\mathrm{Ge}$ nanocrystals. The mean size of the Ge nanocrystals was found to be between 7 and $30 \mathrm{~nm}$ depending on the annealing temperature and duration. With increasing annealing temperatures, defect densities decrease and crystal sizes increase.

Raman scattering was used to monitor the formation of Ge nanocrystals for nitrogen annealed samples. We have demonstrated that in many Raman scattering studies of Ge nanocrystals prolonged annealing results in the formation of $\mathrm{SiGe}$ alloy at the oxide $\mathrm{Si}$ interface. This also results in the detoriation of the Ge nanocrystals in the oxide layers.

Table 1 Measured size of Ge (diamond structure) unit cell.

\begin{tabular}{|c|c|}
\hline Spec. annealing temp. $/{ }^{0} \mathrm{C}$ & cell dimension $/ \AA$ \\
\hline 660 & $5.7 \pm 0.2$ \\
\hline 770 & $5.63 \pm 0.06$ \\
\hline 850 & $5.57 \pm 0.06$ \\
\hline
\end{tabular}

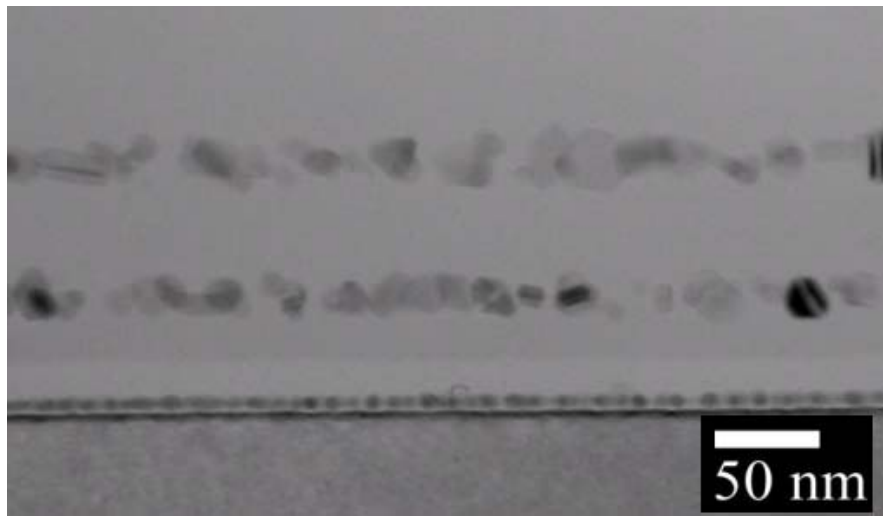

Fig. 1 Cross-sectional TEM micrograph of multilayered sample, grown with $90 \mathrm{sccm}$ of germane flow rate annealed at $900{ }^{\circ} \mathrm{C}$ for 45 min exhibiting Ge nanocrystal bands.
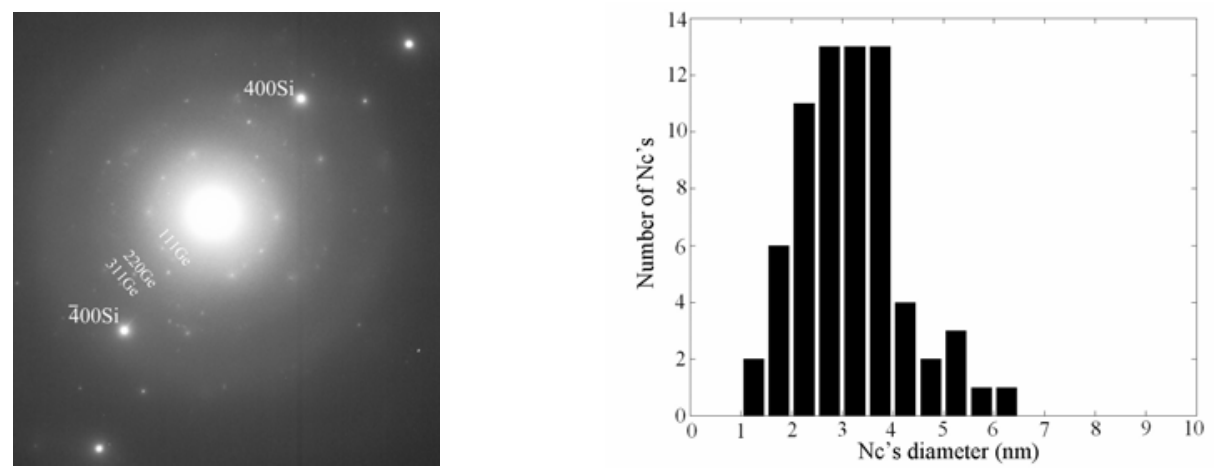

Fig. 2 (a) Typical diffraction pattern for a sample with Ge nanocrystals; (b) shows nanoparticle size distribution in the layers with largest $\mathrm{Ge}$ concentration for the $770{ }^{\circ} \mathrm{C}$ annealing for $300 \mathrm{sec}$. 

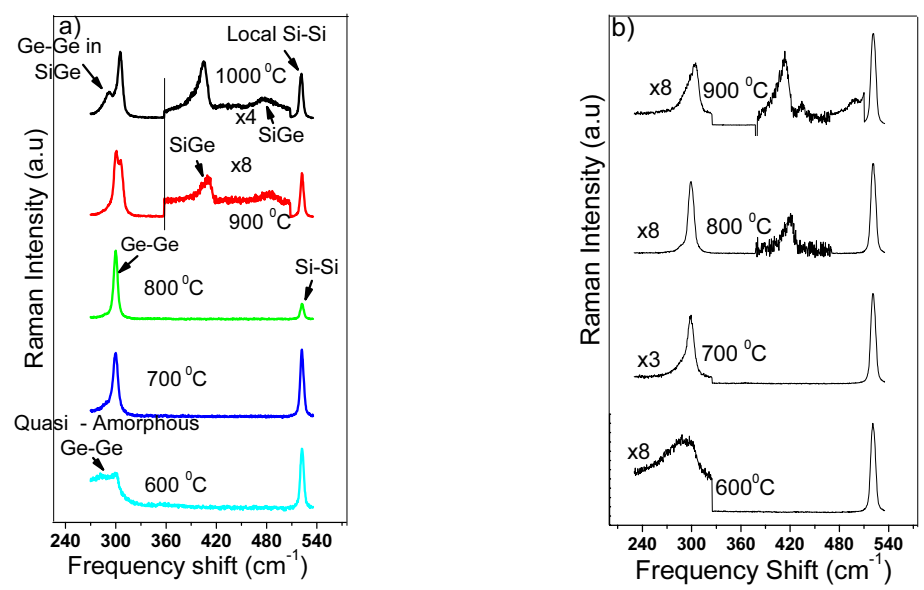

Fig. 3 Raman spectra from $\mathrm{SiO}_{\mathrm{x}}$ : Ge films with $120 \mathrm{sccm}\left(\mathrm{Si}_{1.0} \mathrm{Ge}_{0.67} \mathrm{O}_{3.6}\right)$ (a) and $90 \mathrm{sccm}\left(\mathrm{Si}_{1.0} \mathrm{Ge}_{0.54} \mathrm{O}_{3.4}\right)$ (b) gas flow rate at different annealing temperatures for $45 \mathrm{~min}$.

Acknowledgements This work is a supported by European FP6 project SEMINANO with the contract number 505285 and by TUBİTAK under contract TBAG-U/85(103T115). We gratefully acknowledge Prof. Ş. Süzer of Bilkent University, Chemistry Department for the XPS measurements.

\section{References}

[1] C. Bonafos, B. Garrido, M. Lopez, A. Perez-Rodriguez, J. R. Morante, Y. Kihn, G. Ben Assayag, and A. Claverie, Appl. Phys. Lett. 76, 3962 (2000).

[2] A. Karmous, I. Berbezier, and A. Ronda, Phys. Rev. B 75, 75323 (2006).

[3] U. Serincan, G. Kartopu, A. Guenness, T. G. Finstad, R. Turan, Y. Ekinci, and S. C. Bayliss, Semicond. Sci. Technol. 19, 247 (2004).

[4] R. J. Walters, G. I. Bourianoff, and H. A. Atwater, Nature Mater. 4, 143 (2005).

[5] W. K. Choi, V. Ho, V. Ng, Y. W. Ho, S. P. Ng, and W. K. Chim, Appl. Phys. Lett. 86, 143114 (2005).

[6] Y. Maeda, Phys. Rev. B 51, 1658 (1995). 